\title{
An Urgent Task Ahead
}

EDITORIAL

\section{Maurizio Sabini}

No matter how it has been politically interpreted and used, climate change (and, namely, global warming) is a fact. There is no reasonable (that is "through the tools of reason") denying it and a great deal of data are out there to describe the phenomenon, with its corollary consequence of sea level rising, unfolding under our very eyes. As recently summed up by Ned Cramer from the pages of Architect, if the global average temperature increases more than 3.6 F [2 C] over pre-industrial levels (preventing this, incidentally, is the goal of the 2015 Paris Climate Accord), "there is no stopping the West Antarctic and Greenland ice sheets from melting, which, within this century, could raise the ocean $10 \mathrm{ft}$. [3 m] and $23 \mathrm{ft}$. [7 m], respectively." " With the estimated population of LECZ (low-elevation coastal zones ${ }^{2}$ ) set at about 914 million by 2030 and about 1.2 billion by $2060,{ }^{3}$ the problem for the design and planning fields is serious and solutions are urgently needed.

Of course, for starters, architects, designers, urbanists, private and public developers, organizations and institutions should commit to design and plan buildings, landscapes and human settlements in a way that it does not add to the current, already heavy and vast, global environmental footprint. This would be already quite a seismic paradigm shift from consolidated practices and professional traditions, but it is needed sooner rather than later. As Cramer has admonished, "architecture must change with the climate, and change now, in order for humanity to survive, and hopefully thrive." 4

Yet, countering climate change alone would not be enough. The existing conditions of human settlements at risk due to sea level rising must also be addressed, as we cannot expect to reverse global warming overnight. If resiliency, with Chris Reed, of landscape design firm Stoss, is "the 
ability to adapt to new conditions," 5 it is imperative that as architects, designers and urbanists we rethink our strategies vis-à-vis environments that are rapidly changing for the foreseeable future with more and more people increasingly challenged by the forces of nature. Albert Pope, from Rice University, has aptly observed, while motivating his proposal for Houston of a "tactical retreat" from the 100-year floodplain to make the city more resilient (as a provocative counterpoint to the typical narrative of "advancing"): "we have to get real about the forces that we are contending with, and this will require substantially changing our thinking about advancing." 6

This was the broad framework for the speculative intention of this themed issue of the TPJ. The theme and the questions posed in the call for submissions have been very well received by the global community of scholars, researchers and professionals engaged in research. In fact, the number and the quality of proposals and full submissions have been overwhelming and the selection and review process has not been an easy one. Consequently, we ended up approving an unusually large number of manuscripts, which came to compose a rich, articulated and diverse issue, thus also hoping to meet the expectations of our audience.

Through the issue, one will find a series of different perspectives and lines of inquiry, starting with a reflection, in the form of a "position paper," by TPJ Advisory Board member and urbanist Carlo Gasparrini, on the general meaning of "resilience" and the call for a paradigm shift that this is causing for the design and planning fields.

Then, we offer a few general discussions on design and planning strategies tackling the problem of "(more) resilient edges": from "morethan-resilient infrastructure" (Jefferson \& Lettieri), to "polyvalent adaptation" (Ring), to 3D printing prototyping explorations, such as "habitat grammars" for coastal environments (Van de Riet et al.) and "MaterialNature" coastal protection systems (Farr).

We then decided to group the accepted contributions according to global geographic macro-areas, as the specific conditions of coastal regions and communities around the world have triggered the vast array of inquiries documented in this issue. However, the specificity of environmental, technological, social and cultural conditions notwithstanding, a number of design and planning concepts have emerged with a potential of effectiveness on a more global level. These include: "adaptive strategies" (Leung), "salty urbanism" (Huber et al.), "riskscapes" (Yarina \& Takemoto), "resilient imaginaries" (Gray \& Ocampo), "green infrastructure" (Beja da Costa \& Ribeiro), "selective retreat" (Di Giulio, Lobosco et al.), "multimodal landscapes" (Rossi et al.), "sequential resilience" (Wambecq), and "waterscapes" (Pillen et al.).

Other contributions are more specifically related to their area of study, suggesting nevertheless principles of design intelligence susceptible to be interpreted also within other contexts (Hindle \& Bhatia, Kaprielian, Altschul \& Buchholz, Lee \& Gamez, Firley \& Deupi, McClure, Gamez \& 
Nesbit, Bryant et al., Xiao \& Nguyen, Carlow et al., Clingen \& Bavoso, Multari, and Morcillo Pallares).

Thus, we hope that this issue of the TPJ will serve as a useful reference to all those engaged in investigating on this topic and in testing the applicability and effectiveness of the design and planning ideas here discussed. Coastal and delta regions around the world need and expect our best creativity and intelligence in helping them tackle a problem that is mounting in scope and impact year after year. As Gasparrini observes in his position paper, "awareness is growing not only of what should not be done, but also of what should be done; developing strategies and tactics that are capable of stimulating and governing a resilient metamorphosis of the city."

It is critical that we see this call for resiliency not as a demand for defensive solutions to just fix a problem, but as an opportunity to re-imagine more adaptive environments and a more sustainable world. Henk Ovink, the Dutch special envoy on water affairs, has put it well: "Water is not an enemy. It was never an enemy. It is not a fight, because you will always lose. Resiliency actually means you are not only bouncing back after a disaster, you improve. You bounce back better." 7

Our research challenge and moral obligation are not only to sustain some necessary and fertile lines of inquiry, but also to put our knowledge to test and good use in seizing this opportunity. Starting now, without hesitation.

\section{Notes}

1. Ned Cramer, "So Must Architecture," Architect (October 2017): 138-143 (140).

2. "The LECZ (low-elevation coastal zone) is commonly defined as the contiguous and hydrologically connected zone of land along the coast and below $10 \mathrm{~m}$ of elevation." In Barbara Neumann et al. "Future Coastal Population Growth and Exposure to Sea-Level Rise and Coastal Flooding - A Global Assessment," PLoS One 10, no. 6, published online June 18, 2015. https://doi.org/10.1371/journal.pone.0131375

3. Ibid.

4. Cramer, "So Must Architecture," 143.

5. Chris Reed, as quoted in Mimi Zeiger, "Eye on the Storms," Metropolis (November/ December 2017): 94-99 (98).

6. Albert Pope, as quoted in Zeiger, "Eye on the Storms," 99.

7. Henk Ovink, Special Envoy for International Water Affairs for The Netherlands (and former advisor for the Obama administration), as quoted in "What the Dutch Can Teach the World about Managing Floods," CBC/Radio-Canada, The Sunday Edition with Michael Enright, September 3, 2017: http://www.cbc.ca/radio/thesundayedition/the-sunday-editionseptember-3-2017-1.4265299/what-the-dutch-can-teach-the-world-about-managingfloods-1.4270589 